\title{
THORACOSCOPIC MANAGEMENT OF POSTOPERATIVE ESOPHAGEAL LEAK
}

\author{
Ninh T. Nguyen, MD, David M. Follette, MD, Peter F. Roberts, MD, and James E. Goodnight, Jr, MD, PhD, Sacramento, Calif
}

Esophageal perforation is an uncommon problem, but one that is associated with high mortality $(9 \%-36 \%){ }^{1-3}$ Treatment consists of aggressive surgical management and broadspectrum antibiotics. Surgical approaches to management of esophageal perforation include primary closure, esophagectomy, use of esophageal T-tube, exclusion-diversion, and mediastinal drainage. Selection of treatment depends on factors such as the cause and duration of perforation, clinical condition of the patient, and the degree of surrounding tissue injury.

During the past decade, advances in minimally invasive surgical technology have allowed surgeons to apply thoracoscopic methods to the management of esophageal disease. Thoracoscopic operations have been applied to esophageal myotomy, resection of esophageal diverticulum, excision of esophageal leiomyoma, antireflux operations, and esophagectomy. ${ }^{4}$ In this report we used our experience in video-assisted thoracoscopic operations for the management of 2 patients in whom an esophageal perforation developed as a result of a thoracoscopic esophageal operation.

Clinical summary. The first patient was a 60-year-old woman who was referred with a 2-year history of severe chest pain and intermittent dysphagia. Upper esophageal endoscopy showed no obstructive lesions. Esophageal manometry revealed high-amplitude contraction in the body of the esophagus consistent with a diagnosis of diffuse esophageal spasm. The patient was treated nonoperatively with calcium-channel blockers and nitrates without success. She was offered surgical treatment consisting of a long esophagomyotomy. The operation was performed through the right side of the chest with 4 thoracoscopic trocars. Intraoperative endoscopy after the myotomy revealed no air leaks (Fig 1). The patient was discharged on postoperative day 4 on a clear liquid diet.

On postoperative day 7 , the patient returned with shortness of breath and acute chest pain. Gastrograffin contrast study revealed extravasation of contrast material at the midesophageal region. Endoscopy showed a small 3-mm perforation at the middle esophagus. The patient was reexplored thoracoscopically. Intraoperative endoscopy identified the

From the Department of Surgery, University of California, Davis, Health System, Sacramento, Calif.

Received for publication June 29, 2000; accepted for publication July 18, 2000.

Address for reprints: Ninh T. Nguyen, MD, Department of Surgery, 2221 Stockton Blvd, 3rd Floor, Sacramento, CA 95817-1418 (Email: ninh.nguyen@ucdmc.ucdavis.edu).

J Thorac Cardiovasc Surg 2001;121:391-2

Copyright () 2001 by The American Association for Thoracic Surgery

$0022-5223 / 2001 \$ 35.00+0 \quad \mathbf{1 2 / 5 4 / 1 1 0 4 8 5}$

doi: $10.1067 / \mathrm{mtc} .2001 .110485$

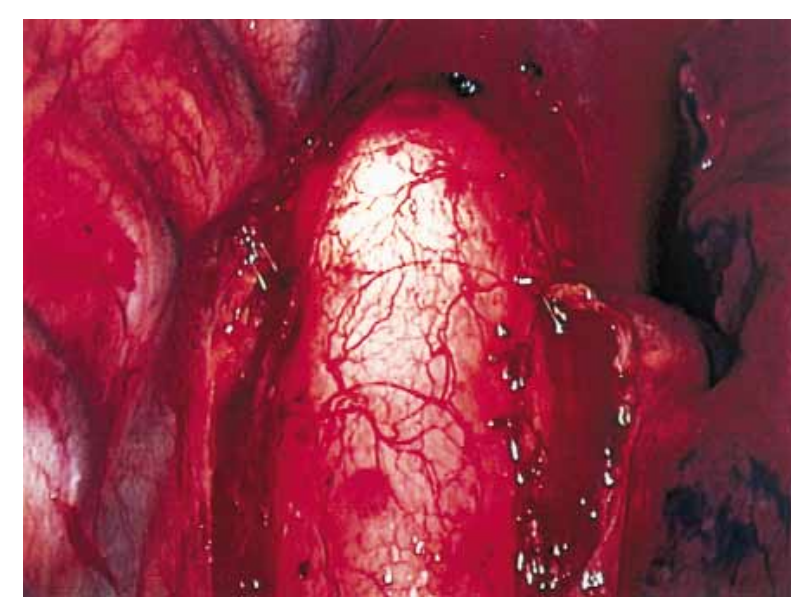

Fig 1. Thoracoscopic long esophagomyotomy with intraoperative endoscopy.

perforation (Fig 2). Fibrinous exudate overlying the esophagus was debrided, and the perforation was closed thoracoscopically with interrupted sutures.

The second patient was an 83-year-old man with a 3-month history of dysphagia. Upper esophageal endoscopy revealed a gastroesophageal junction tumor with extension into the gastric cardia in the setting of Barrett esophagus. The patient underwent an uncomplicated laparoscopic and thoracoscopic Ivor Lewis esophagectomy with an intrathoracic stapled anastomosis. Initial gastrograffin contrast study demonstrated no leak. On postoperative day 13, he was discharged.

On postoperative day 21 , he was readmitted for acute chest pain and shortness of breath. The chest x-ray film revealed a large, right-sided pleural effusion. Upper esophageal endoscopy revealed an anterior perforation at the anastomosis. The patient was reexplored through the right side of the chest by means of 4 thoracic trocars. The lung was retracted medially to expose the gastric conduit, and intraoperative endoscopy with insufflation revealed an air leak at the anastomotic line. A loop wire was inserted transthoracically through the perforation defect into the gastric conduit. Endoscopy was used to retrieve the loop wire, which was then brought out through the mouth. The end of a T-tube was sutured to the loop wire, the wire was pulled, and the T-tube was dragged transorally through the right side of the chest until its tip was positioned intraluminally at the perforation site. The position of the T-tube was confirmed by endoscopy. A Jackson-Pratt drain was placed next to the site of perforation. Two 32F chest tubes were placed for postoperative drainage.

Postoperative courses. Patient 1 did well postoperatively. A contrast study performed 7 days after the repair revealed no 




Fig 2. Thoracoscopic view showing site of esophageal perforation (arrow).

leaks. The patient was discharged on postoperative day 9 , with a Jackson-Pratt drain in place. The drain was subsequently removed 3 weeks after the operation.

Patient 2 recovered and was discharged to a rehabilitation facility on postoperative day 28 with a Jackson-Pratt drain and esophageal T-tube in place. He was started on clear liquids at 6 weeks after discharge. The esophageal T-tube was slowly withdrawn each week over a period of 4 to 6 weeks.

Comments. Perforation after a transthoracic esophageal operation is a life-threatening condition. The primary symptoms are acute chest pain, shortness of breath, and fever. Early recognition and definitive surgical intervention are important to control sepsis. The management consists of surgical debridement, control of the perforation site (primary closure or esophageal T-tube placement), and appropriate antimicrobial therapy.

Thoracoscopy for management of esophageal perforation is limited. Kiel and colleagues ${ }^{5}$ reported the case of a patient who had an esophageal perforation repaired with a thoracoscopically assisted procedure through a $5-\mathrm{cm}$ thoracic incision to directly suture the esophageal defect. Laisaar ${ }^{6}$ reported on 2 patients with esophageal perforation who were treated with thoracoscopic drainage of pleural empyema. The sites of perforation were not identified, and these patients were treated with pleural drainage alone.

In our limited experience with 2 patients, the goals for minimally invasive treatment of esophageal perforations were to (1) identify the site of esophageal perforation, (2) control the esophageal leaks (primary closure or T-tube placement), (3) debride necrotic debris, and (4) drain the mediastinum. Our thoracoscopic approach required 4 thoracic trocars. The initial step after insertion of the camera was to evacuate fibri- nous debris and purulent pleural exudate. We exposed the esophagus by retracting the lung medially.

Intraoperative endoscopy was used to identify the site of perforation. The suspected area of perforation was submerged under water during endoscopic insufflation to identify the perforation. Air bubbles revealed the perforation in both patients. Once the perforation site was identified, the treatment depended on the degree of surrounding tissue injury and the clinical condition of the patient. In the first patient the esophageal defect was identified to be small (2-3 $\mathrm{mm}$ ), in the midportion of the esophagus, and surrounded with viable tissue. A primary closure of the defect was performed with interrupted sutures. In the second patient an anastomotic leak was identified as a defect of 4 to $5 \mathrm{~mm}$ in diameter surrounded by severely inflamed tissue. We placed a T-tube across the perforation site in this patient. Our method for placement of the T-tube was similar to the pull-through method for placing a percutaneous gastrostomy tube; it was simple and did not require enlargement of the perforation defect. The correct position of the T-tube was confirmed with intraoperative endoscopy.

Esophageal perforation is a complex problem with high surgical risks. Surgical drainage and control of the leak continue to be the mainstays of treatment. In selected cases of esophageal perforation, thoracoscopy can be applied by using the same surgical principles as in the open thoracotomy approach. Advantages of the video-thoracoscopic approach are the excellent view of the whole thoracic cavity and adequate debridement and proper drainage of all pleural cavity areas. Either primary closure or an esophageal T-tube can be used to close the esophageal leak. By avoiding a major thoracotomy, the patient benefits from less postoperative pain, a decrease in wound-related complications, and a faster postoperative recovery.

\section{REFERENCES}

1. Bufkin BL, Miller JI Jr, Mansour KA. Esophageal perforation emphasis on management. Ann Thorac Surg 1996;61:1447-51.

2. Attar S, Hankins JR, Suter CM, Coughlin TR, Sequeira A, McLaughlin JS. Esophageal perforation: a therapeutic challenge. Ann Thorac Surg 1990;50:45-9.

3. Reeder LB, DeFilippi VJ, Ferguson MK. Current results of therapy for esophageal perforation. Am J Surg 1995;169:615-7.

4. Luketich JD, Nguyen NT, Weigel T, Ferson P, Keenan R, Schauer P. Minimally invasive approach to esophagectomy. J Soc Laparoendosc Surg 1998;2:243-7.

5. Kiel T, Ferzli G, McGinn J. The use of thoracoscopy in the treatment of iatrogenic esophageal perforations. Chest 1993;103: 1905-6.

6. Laisaar T. Video-assisted thoracoscopic surgery in the management of acute purulent mediastinitis and pleural empyema. Thorac Cardiovasc Surg 1998;46:51-4. 